\title{
Beiträge zur Anwendung der Massage in der Augenheilkunde.
}

\author{
Von \\ Dr. Conrad Dantźiger, \\ pract. Arzt.
}

Die wenig zahlreichen Angaben, welche in der Literatur über die Anwendung der Mássage in der Augenheilkunde vorliegen, sind sämmtlich neueren Datums.

Das Verdienst als erster auf die Brauchbarkeit der Massage in der Augenheilkunde hingewiesen zu haben, gebührt Donders*), welcher auf dem internationalen ophthalmologischen Congress zu London im Jahre 1872 das sogenannte Traitement de massage, die Behandlung durch Kneten erwähnt und den Versuch dieser Behandlungsweise bei Krankheiten der Cornea empfehlen zu dürfen glaubt. Diese Empfehlung des berühmten Gelehrten fand augenscheinlich damals wenig Gehör: die Literatur der nächsten Jahre weist nur eine Arbeit von Heiberg**) auf, welcher bei Flecken und Facetten der Hornhaut die Massage in Anwendung zog und speciell bei den Hornhautflecken durch die Massagebehandlung eine Besserung der

*) Zehender's Monatsblätter, 1872, p. 302.

**) Nagel's Jahresberichte, 1874, p. 268. 
Sehschärfe von $\frac{20}{200}$ (Snellen's Tafel) auf $\frac{20}{50}$ (desgl.) erzielte; die Behandlungsdauer betrug oft Monate. - Eine kurze Arbeit über die Massage des Auges giebt im Jahre 1878 Pagenstecher*): P. berichtet über drei mit Massage erfolgreich bebandelte Fälle, von denen der erste um deswillen besonders interessant ist, weil sioh bei demselben constant nach der Vornabme der Massage eine nicht unerhebliche Verminderung des intraocularen Drucks ergab. Gradenigo**) knüpft an diese von Pagenstecher beobachtete Verminderung des intraocularen Drucks an und berichtet, dass die Abnahme der Tension bei der Mussage am gesunden Auge schon nach 2-6 Minuten wahrnohmbar ist; wie Schenkl***) mitheilt, haben Wicherkiewicz und Schnabel die Tensionsabnahme durch Massage des Auges in rollem Masse bestätigt und sodann bei Glancom die Massage versucht: Es ergab sich constant, aber nur temporär beim Glaucom eine Verminderung des intraocularen Drucks. Chodin†) versuchte die Massage des Anges nach Discisio cataractae behufs Beschleinigung der Resorption der Linse mit sichtbarem Erfolge; er erklärt sich hierbei die Wirkung der Massage rein mechanisch, der Art, dass das Kneten in der Sceralgegend die Linsensubstanz in die Vorderkammer hervordrängt und dass das Kneten in der Hornhautgegend die vorgefallene Linsensubstanz "zerdruckt und zermalme".

Weitere Beiträge zur: Massage des Auges lieferten Pagenstecher, Pedraglia, Just, Damalix, van der Laan. p. 281 .

*) Hirschberg, Centralbl. für practische Augenheilk., 1878,

***) Hirschberg, Centralb1, fur practische Augenheilkunde, 1880, p. 123.

***) Prager, medicinische Wochenschrift, 1882, No. 29.

†) Hirschberg, Centralblatt für practische Augenheilknnde, 1880, p. 279. 
Pagenstecher*) hält in erster Linie die Trübungen der Cornea als für die Massagebehanlung geeignet: der von ihn mitgetheilte Fall ist allerdings im hohen Masse passend, die Wirkungsfähigkeit der Massagetherapie zu illustriren. Es gelang nach 25 maligem Massiren ein Auge, welches mit seit 20 Jahren bestehenden, ausgedehnten Hornhauttrübungen behaftet einen Visus von $5 / 100$ (Snellen'sche Tafel) hatte, so weit zu fördern, dass ein Visus von $5 / 30$ (desgl.) sich darbot. Von Conjunctival-Erkrankungen hält Pagenstecher für die Massagebehandlung geeignet die Conjunetivitis phlyotaenulosa und den sogenannten Frühjahrskatarrh; zu den Soleral-Affectionen übergehend bespricht Pagenstecher die geradezu überraschenden Heilerfolge der Massage bei acuter and chronischer Episcleritis; weiterhin erwähnt er einen Krankheitsfall, den er als circumscripte Entzündung des Corpus eiliare deutet, welcher mit Massage erfolgreich behandelt wurde. Pedraglia**) massirte mit gutem Resultat in zwei Fällen von ganz frischer Epi= scleritis, Ju st***) versuchte die Massagebehandlung in einem Fall von Hypopyon und erzielte eine überraschend schnelle Heilung. Genaueres casuistisches Material liefert Damalix $\uparrow$ ). Von den sieben von ihm mitgetheilten Fällen sind die drei ersten scheinbar dem Werthe der.Massage des Auges ungünstig: es handelte sich nämlich zunächst um zwei Fälle von alten Granulationen nebst consecutivem Pannus, gegen welche Affectionen alle nur irgendwie sonst angewandten Mittel ohnmächtig gewesen waren and die einer täglichen Massagebehandlung unterworfen absolut sich nicht besserten. Derartige Fälle gehören nach meinem Dafürhalten überhaupt p. 225 .

*j Knapp und Hirsch berg, Archiv für Augenheilkunde, 1881,

**) Hirschberg, Centralbl. für practische Augenheilk., 1881, p. 111.

***) Daselbat, p. 173.

†) Archives d'Ophthalmologie. I. 6. 
nicht in die Grenzen dex Indicationen für die Massage und können wohl kaum die sonst vielfach erprobte Leistungsfähigkeit der Massage des Anges entkräften. Der dritte Fall von Damalix ist insofern erwähnenswerth, als dieser Kranke nach Vornahme der Massagebewegungen stets so heftige Reizerscheinungen bekam, dass die Massage völlig bei Seite gelegt werden musste. Sehr günstig verliefen dagegen unter der Massagebehandlung drei Fäle von parenchymatöser Keratitis bei jugendlichen Individuen und auch ein Fall pon Granulationen mit consecutivem Pannus bei einem 13 jährigen Mädchen bot einen guten Erfolg dar. Van der Laan massirte bei traumatischer Cataract und beobachtete in diesem Falle durch die Massage das Verschwinden der glaucomatösen Erscheinungen. Aus neuester Zeit sind von Beiträgen zur Massage des Auges solche ron Klein, Sohenkl, Carré, Friedmann, Heisrath, Hock, Gradenigo zu registriren.

Klein*) referirt uber gute Erfolge der Massage bei friseher Episcleritis, bei phlyetänulärer Ophthalmie, diffuser parenchymatöser Keratitis und beim Frühjahrskatarrh; er rommt auf die druckmindernde Wirkung der Massage zurück und empfiehlt beim absoluten Glaucom und bei den der Operation nioht mehr zugänglichen Glaucomfällen den Versuch mit der Massage. Schenkl**) massirte bei zwei frischen Hornhauttrübungen mit überraschendem Erfolg, er ist in der Lage sodann ein Analogon za Pagenstecher's erfolgreich massirtem Fall von alter Hornhautträbung zu liefern, indem er die Krankengeschichte eines 46 jährigen Tagelöhners mittheilt, der seit fruhester Jugend mit ausgedehnten maculösen Hornhauttrübungen behaftet einen Visus

*) Wiener medicinische Presse, 1882, No. 9, 10, 12, 15 .

*) Prager, medicinische Wochenscbrift, 1882, No. 29 31 und 32 . 
R. A. $=\frac{20}{100}$ (Snellen'sche Tafel) L. A. $=\frac{20}{1000}$ (desgl.) hatte und schon nach achttägiger Massage einen Visus R. A. $=\frac{20}{100}$ (Snellen'sche Tafel) L. A. $=\frac{20}{70}$ (desgl.) erkennen liess. Eine ca. 1/1/2 Jahre bestehende maculöse Hornhauttribung eines jugendlichen Invidiums wurde von Schenkl durch zehimalige Massage vollig beseitigt. Günstig verliefen auch bei der Massagebehandlung fünf Fälle von parenchymatöser Keratitis, sodann einige Fälle von Episcleritis und von Frühjahrskatarrh; er referirt sodann über zwei Fälle von chroniseher recidivirender Iritis, von denen der eine die Massage wohl vertrug, aber nicht gebessert wurde, während in dem zweiten ein im Entstehen begriffenes Iritisrecidiv durch die eingeleitete Massage geradezu coupirt wurde. Die Versuche dieses Autors bei Hypopyon, bei den versehiedenen Formen des Glaucoms sind nicht günstig, nur in einem Fall von Secundärglaucom erzielte die zehnmalige Massage dauernde Herabsetzung des intraocularen Drucks und Nachlassen der Schmerzen und Reizerscheinungen. Carré $e^{*}$ berichtet aber Massageversuche bei Keratitis parenchymatosa resp. interstitialis, bei Keratitis phlyctänulosa und bei Blepharitis ciliaris und urtheilt uber die Erfolge sehr günstig; Friedmann**) behandelte 15 Augen mit Massage und theilt einen besonders erfreulichen Fall von nicht mehr friseher Hornhauttrubung mit, der nach vierwochentlicher Massage völlig geheilt wurde. Hock ${ }^{* * *}$ ) erlebte dagegen bei einer mit Massage behandelten Hornhauttrübung einen völligen Misserfolg, Gradenigo $\dagger$ ) sah unter der Massage

*) Gazette d'Ophthalmologie, 1882, No. 9.

**) Wiener medicinische Presse, 1882, No. 23.

***) Wiener medicinische Blätter, 1883, No. 12.

t) Hirschberg, Centralblatt für practische Augenheilkunde, 1883, p. 573. 
einen Fall von Tenonitis schnell heilen. Heisrath*) ist in der Lage über 400 Augen mit granulöser Bindehautentzündung zu berichten, die massirt ein-sehr befriedigendes Resultat ergaben. Nur jene Fälle von Bindehautgranulationen hält Heisrath für die Massage passend, welche das acute Stadium überwunden and noch keine secundären Veränderungen an Conjunnctiva und Tarsus aufweisen. Während meiner sich ca. auf $1 / 2 \mathrm{Jahr}$ erstreckenden täglichen Besuche der Heisrath'schen Poliklinik hatte ich rollauf Gelegenheit, die wirklich grosse Leistungsfähigkeit in solchen Fällen von Granulationen zu sehen und bin in der Lage, hierfur die Massage warm zu empiehlen. Handelt es sich um alte Granulationen mit schweren consecutiven Veränderungen an Conjunetiva, "Tarsus und Cornea, so nützt vor der Hand die Massage verschwindend wenig. Hier gilt es, zunächst die erkrankten resp. degenerirten Bindehautparthieen fortzuschaffen - dieser Indication entspricht die von Heisrath empfohlene Excision der Granulationen - und damn den spontan nioht recht riuckbildungsfähigen, restirenden Hormhautveränderungen therapeutisch zu begegnen. Soweit diese Consecutivveränderungen der Hornhaut sich als Trübungen präsentiren, liegt nun, wie ich in dem zweiten Theil der Arbeit darthuen werde, in der Massage eine vortreffliche Behandlungsmethode vor.

Beror ich die Technik der Massage des Auges bespreche, erwähne ich noch eine Operation, die in einigen der von mir massirten Hornhauttrubungen der Massage vorausgeschickt wurde und welche in dieser Weise mit Massage combinirt einen entschiedenen Fortschritt in der Behandlung speciell alterer Hornhauttrubungen bedeutet. Die Operation, gewöhnlich Abrasio corneae genannt, und ihr therapeutischer Zweck - die Aufhellung von Corneal-

*) Hirschberg, C. f. p. A., 1883, p. 412. 
trübungen - ist nicht neu. Das Verdienst jedoch, die Abrasio corneae in Combination mit nachfolgender Massage bei der Behandlung von Hornhauttrübungen erprobt $z a$ haben, gebührt Heisrath, dem ich auch meine Erfahrungen über diesen Gegenstand verdanke.

Die Abrasio corneae, schon im vorigen Jahrhundert gekannt und versucht, hat sich in der operativen Augenheilkunde nie ein eigentliches Bürgerrecht erwerben können; abgesehen von den bisweilen nur sehr mässigen Heilerfolgen derselben kann dieselbe ernste Gefahren. für das operirte Auge involviren. In früheren, vorantiseptischen Zeiten hat man suppurative Keratitis, Iritis, ja selbst Panophthalmitis nach der Operation sich entwickeln sehen und man darf wohl sagen, dass bei Berücksichtigung solcher Eventualitäten die Abrasio corneae ein ultimum refugium für ganz verzweifelte Fälle darstellen musste. Heute, wo wir auch in der Augenheilkunde antiseptisch operiren, steht die Sache etwas anders. Ich sah die Operation wiederholt ausführen und beobachtete häufig den Verlauf nach derselben und weiss von keinem Fall, der verhängnissvoll verlaufen ist. Freilich wurde stets streng antiseptisch operirt und desgl. in der Nachbehandlung verfahren. Da die Operation sehr schmerzhaft ind subtil ist und der Bulbus gut fixirt sein muss, erseheint es unbedingt nöthig, zu narkotisiren; in den zuletzt operirten Fullen wurde zur Erzielung einer localen Anaesthesie eine 3 proc. CocaïnLösung verwandt, von der 10 Tropfen völlig dem gewünschten Zweck entsprachen und die Chloroformnarkose entbehrlich machten. Man kann die Abrasio corneae auf zweifache Art ausführen, entweder man schabt-oder kratzt mit der Schneide eines scharfen Messers das Epithel und die oberflächlichen. Hornhautschichten ab oder.man trägt das Cornealgewebe in zusammenhängenden Stücken oder Schollen $a b$; die von mir beobachteten Fälle wurden stets auf die erstere Art operirt und zum Abkratzen das จ. Graefe's Arehiv fur Ophtbalmologie XXXI. 3. 
Graefe 'sche Staarmesser gebraucht. Das eigentliche Abschaben resp. Abkratzen geschah genau so, als wenn man einen Tintenfleck auf Papier mit der Schneide eines Messers ausradiert; selbstverständlich wurde jeder stärliere Drick möglichst vermieden und das Abkratzen nar auf das direct veränderte Cornealgewebe besehränkt. Der Verlanf nach der Operation ist ungefähr der folgende: Das nach der Operation dem Patienten stark empfindliche Auge zeigt nach 24 Stunden bei Abnahme des Verbandes ziemlich starke Reizerscheinungen. Im Bereiche der abgekratzten Parthe, nicht selten aber auch uber diese hinaus constatirt man eine frische, des Epithels verlustige Infiltration der Cornea von sehmutzig graner Farbe. Am besten hütet der Operirte 2-3 Tage das Bett, macht 2-3 Mal tüglich warme Umscbläge und erhält regelmässig wegen der eventuellen GeÎahr einer iritischen Reizung Atropineinträufelungen. Schon am zweiten oder dritten Tage nach der Operation ziehen Gefässe über den Corneoscleralrand hin zu dem frischen Infiltrat: man hat im wesentlichen das vor sich, was man einen Pannus regenerativus nennt. Die Reizerscheinungen lassen gewöhnlich zwischen dem fünften und dem achten Tage nach, bis zu welcher Zeit sich auch die Regeneration des Epithels rollzogen hat. Nun ist es an der Zeit, die Massage zu beginnen, wobei joh noch bemerke, dass vereinzelte, zu dem Infiltrat ziehende Gefässe, da sie bald verôden, die Massage nicht contraïndiciren.

Theoretisch denkt man sich die Wirkung der Abrasio corneae so, dass auf derartige Weise gesetzte Substanzverluste in einzelnen Fallen durch neugebildetes Gewebe wieder ausgeglichen werden, welches in Bezug auf Durchsichtigkeit der normalen Hornhautsubstanz nahekommt. Würde die Wirklichkeit dieser Theorie voll entsprechen, so ware die Abrasio corneae - zumal unter antiseptiscnen Kautelen ausgefuhrt - eine geradezu ideale Behandlungsart für Hornbauttrübangen. Leider ist nun aber der 
unmittelbare Effect der Operation meist kein sehr schöner: das frische Infiltrat ist fast stets trüber als die vor der Operation bestehende Trübung und auch der Visus ist selbst bei geringen Reizerscheinungen meist geringer als vordem; die Massage des Auges bietet nun, wie es scheint eine treffliche Handhabe, um diese frisch gesetzten Infiltrationen bis zu einem gewissen Grade aufzuhellen.

Ich gehe nun dazu über, einiges ubber die eigentliche Technik der Massage des Auges zu berichten, und erörtere zunächst die Frage: Soll man trocken oder mit Verwendung irgend eines Fettes oder einer medikamentösen Salbe massiren? Heisrath liess stets mit Salbe massiren, andere Autoren wie Klein, Pedraglia, Carré liessen die Salbe fort und massirten trocken; Klein z. B. liess die Salbe fort, um eine reine, ungetrübte Beobachtung der Massagewirkung zu haben und die Massage liess sich nach seinen Erfahrungen auf diese Art gut und schmerzlos ausführen und gab sehr gute Heilresultate. Damalix, Sohenkl bedienten sich stets zur Massage einer Salbe, auch Pagenstecher kam von der anfünglich geübten trockenen Massage $a b$ und wandte späterhin stets eine Salbe an. Pagenstechers Gründe für den Gebrauch einer Salbe bei der Massage des Auges sind einfach und plausibel: die Massagebewegungen sind eben leichter and besser ausführbar, wenn der Bulbus leicht eingefettet ist, sodamn erscheint es aber auch zweifellos, dass die Wirkung gewisser Salben durch die Massage mit denselben erhöht wird. Die von Pagenstecher zumeist benutzte Salbe war die gelbe Präcipitatsalbe, welche er in 1-10 Procent Mischung mit Vaseline als Vehikel verwandte; zu meinen Massageversuchen wurde stets eine gelbe, folgendermassen formulirte Salbe gebraucht: Hydrargyr. praecipitat. flav. 0, 1.

Vaseline 6,0. - Eine andere, von Heisrath empfohlene und rielfach zumal bei Granulationen erfolgreich gebrauchte Salbe ist formulirt: 
Kal. jodat. 0,3 .

Natr. bicarbonic. 0,25.

Vaseline 5,0 .

In Betreff der eigentlichen Massagebewegungen räth Pagenstecher, dass man, ,mit dem Daumen oder Zeigefinger das obere oder untere Lid in der Nähe des Lilurandes erfasst und dann vermittelst des Lidrandes selbst Reibungen auf dem Auge, und zwar möglichst schnell vornimmt". Heisrath låsst in einer von Pagenstecher etwas abweichenden Art massiren: nachdem der Zeigefinger der rechten Hand soweit mit gelber Salbe versehen ist, dass die Fingerkuppe gerade einen leichten Fettglanz zeigt, wird das untere Lid des zu massirenden Auges mit einem Finger der anderen Hand leicht herabgezogen und dann die Salbe vorsichtig der Art in den unteren Conjunctivalsack gebracht, dass der eingefettete Finger die Salbe am Lidrande abstreicht; Patient wird behufs besserer und allgemeiner Vertheilung der Salbe auf dem Bulbus angewiesen, die Lider zu schliessen. Hierauf legt man den Zeigefinger der rechten Hand auf die Mitte des oberen Augenlids and führt leichte, reibende oder vielmehr zitternde Bewegungen mit dem Lide aus, bei denen das obere Lid meist von rechts nach links uber den Bulbus bewegt wird; die Massage wird tăglich einmal vorgenommen, jede Sitzung danert ca. eine halbe Minute. Ich bemerke noch, dass jedes Drücken, Kneten, Klopfen u. s. w. wie bei der Massage an anderen Körperstellen ublich und zulässig bei der Massage des Auges absolut unstatthaft ist. Es handelt sich um ein methodisches, sanftes Reiben, bei dem, der Finger mit dem oberen Lid förmilich über den Bulbus dahinfliegen muss". - Nach der jedesmaligen Massage tritt eine leichte Röthung des Anges ein, die, wenn auch meist schnell vorübergehend, es praktisch erseheinen lässt, den Patienten noch 10-15 Minuten nach Vornahme der Massage im Zimmer warten zu lassen. Tritt nach der Massage ein 
heftigerer, constant länger als eine halbe Stande dauernder Reizzustand des Auges ein mit starkem Thränenträufeln und Schmerzen, só müssen wir die Massage aussetzen, bisweilen völlig ruhen lassen. Grosse Vorsicht mit der Massage ist dann nöthig, wenn eine iritische Reizung oder selbst Iritis vorliegt, weil sie dann nach unseren Beobachtungen sehr angünstig wirkt. -

Ich schliesse nunmehr die Mittheilung der von mir unter Heisrath's Leitung massirten Fälle von Hornhauttrubbungen an; es handelt sich um zehn Fälle von Hornhauttrübungen, von denen sechs mit einfacher Masșage unter Benutzung der gelben Salbe, vier mit Abrasio corneae und nachfolgender Massage behandelt sind. Zur Bestimmung der Sehschärfen benutzte ioh die Snellen'sche Tafeln und die Jäger'schen Schriftproben, in jedem Falle wurde der Effect dieser Untersuchungsmethoden durch das Verhalten von Brillengläsern controlirt und eventuell verrollständigt.

I. E. Wars chun, scrophulöser junger Mann; auf dem linken Auge, das zuerst und sehr intensiv erkrankt war, bestehen seit ca. acht Jahren Hornhautflecken, in und um welche sich in den letzten vier Jahren wiederholt alle drei bis vier Wochen leichtere Hornhaut-Entzündungen etablirt haben, die constant die Sehschärfe des Auges verschlechterten. Die Hornhautflecken des rechten Auges bestehen seit einem Jahre.

25. Juni 1884. Status. R. Auge: Cornea ungefähr $2 \mathrm{Mm}$. nach unten aussen vom Hornhautcentrum besteht ein gleichmässig grauweisser Flecken, leicht durchscheinend, ca. $1 \mathrm{Mm}$. von rechts nach links breit, ca. $2^{1 / 2} \mathrm{Mm}$. von oben nach unten lang; nach innen unten $1 \frac{1}{2} \mathrm{Mm}$. vom Hornhautcentrum besteht eine punktförmige, grauweisse Trübung. 
Status. L. Auge: die Cornea in ihrer unteren Hälfte intensiv weiss getrübt in einer Ausdehnung von ca. $3 \mathrm{Mm}$. ron rechts nach links lang, ca, $2 \mathrm{Mm}$. von oben nach unten breit; die obere Hornbauthalfte ist Sitz einer diffusen, leicht graulich gefarbten, ca. $3 \mathrm{Mm}$. Flächendurchmesser habenden Trübung.

Visus. 25. Juni 1884. R. Auge $=2 / 4($ fast $2 / 3$ ) der Snellen'schen Tafeln; Jäger Nr. 1. 6 Zoll Entfernung.

L. Ange. Visus $=\frac{20}{200}$ (Snellen). Jäger 13. 5 Zoll Entfernung.

25. Juni 1884 Beginn der Massagebehandlung.

20. Juli 1884. R. Cornea: der unten aussen gelegene Wlecken ist in seiner Peripherie merklich aufgehellt und von mattgrauer Farbe; der punktförmige Flecken ist unverändert.

L. Cornea: keine Besserung.

20. Juli 1884. Visus. R. Ange $=2 / 3$ (Snellen). Jäger 1. 6 Zoll Entfernung.

L. Auge $=\frac{20}{200}$ (Snellen). Jäger 11. 5 Zoll Entfernung.

Patient wird in seiner Heimath weiter massirt und stellt sich vor am 17. October 1884 .

17. Oetober 1884. R. Cornea: Der unten anssen gelegene Fleck beinahe völlig anfgehellt, dor punktformige unverăndert.

I. Cornea: nichts deutlich in der Intensitat der Flecken gebessert.

17. October 1884. Visus, $r$. Auge $=1$ (Snellen).

Jäger 1. 6 Zoll Entfernung.

L. Auge $=\frac{20}{100}$ (Snellen). 


\section{Jäger 6. 5 Zoll Entfernung.}

Das linke, in den letzten vier Jahren immerfort von Recidiven heimgesuchte Auge ist seit Vornahme der Massage völlig von Rediciven frei; eine nochmals, am 15. Januar 1885 vorgenommene Untersuehung bestatigte den Visus rom 17. October 1884 völlig und ergab auch das weitere Befreitsein des linken Auges von Rediciven.

II. R. Heling, 13 Jahre alt, seit 31/2 Jahren schwere Trübung der rechten Cornea; Grundleiden: Scrophulose.

29. September 1884. Status, R. Auge. Cornea: ziemlich in der Mitte derselben besteht eine intensive, leucomatöse Trübung, nur schwach durchscheinend, von nierenförmiger Gestalt, ca. $6 \mathrm{Mm}$. von oben nach unten lang, ca. $3 \mathrm{Mm}$. von rechts nach links breit; nach innen von derselben eine ca. $1 \mathrm{Mm}$. Flächendurchmesser habende, grauweisse, durchscheinende Trübung.

29. September 1884. Visus, r. Auge = Finger gezählt auf 20 Fuss Entfernung. Kein Jäger.

2. Ootober 1884. Abrasio corneae in Chloroformnarcose mit dem Graefe'schen Staarmesser; es wird nicht nur das Hornhautepithel, sondern auch das Hornhautgewebe oberflächlich abgekratat. Jodoform, Einträufelung von Atropin, Verband, Bettruhe.

8. October 1884. Die nach der Operation eingetretenen Reizerscheinungen sind geschwunden, das abgekratzte Epithel ersetzt; es besteht eine diffuse, graue Trübung der Cornea, mässig durchscheinend.

8. Oetober 1884. Beginn der Massage.

8. Norember 1884. Sehr ausgiebige Aufhellung: die vor der Operation vorhandene, nach innen gelegẹne Trübung ist total beseitigt, das an Stelle der leucomatösen Trübung durch die Abrasio gesetzte Infiltrat ist graulich und von mässiger Intensität. 
8. November 1884. Visus, r. Auge $=\frac{20}{70}$ (Snellen). Jäger 3. 6 Zoll Entfernung.

Leider konnten weitere Erhebungen nicht gemacht werden, da Patient seine Heimath wieder aufsuchen musste.

III. Pridde, 22jähriger junger Mann, im Jahre 1881 an beidersoitiger Granulose erkrankt; das linke, ersterkrankte Auge wurde 1881 in kürzester Zeit von den Granulationen befreit, das rechte Auge erkrankte schwerer und es entwickelte sich schnell ein. Pannus crassus, der trotz beständiger Behandlung sich nichtrührte. Im September 1884 Excision der granulös veränderten Bindehautparthien und des Tarsus superior, darauf Patient nach ca. 5 Wochen von den Bindehautgranulationen and dem pannus crassus geheilt. Zur Aufhellung der restirenden Hornhauttrübungen wird am 18. October 1884 die Massagebehandlung eingeleitet.

18. October 1884. Status, i. Cornea ist in ihren beiden oberen Dritteln Sitz einer intensiven, graulichen Trübung; innerhalb dieser getrübten Parthie lässt sich noch eine intensivere, grauweisse, wenig durehscheinende Trübung abgrenzen, die bis zum Hornhantcentrum herabreicht and ron oben nach unten ca. $3 \mathrm{Mm}$. lang und ron rechts nach links ca. $1 \mathrm{Mm}$. breit ist.

Visus, r, Auge $=$ Finger gezählt auf 6 Fuss Entfernung. Kein Jäger.

1. November 1884. Nach 14tăgiger Massage Trübung sichtlich heller geworden.

1. November 1884. Visus, r. Auge = Finger gezühlt auf 15 Fuss Entfernung. Kein Jäger.

25. Januar 1885. Visus, r. Ange $=$ Finger gezählt auf 20 Fuss Entfernung, Jager 12. 5 Zoll Entfernung. 
12. März 1885. Visus, r. Auge $=\frac{20}{200}$ (Snellen).

Jäger 8. 6 Zoll Entfernung.

30. März 1885. Visus, r. Auge $=\frac{20}{200}$ (Snellen).

Jäger 7. 6 Zoll Entfernung.

30. März 1885. Status. Die Aufhellung der Trübungen ist deutlich constatirbar; besonders ist jene Trubung, die sich als grauweisse und wenig durchscheinende innerhalb der diffusen, graulichen Parthie abhob, zusehends aufgehellt und hebt sich kaum noch von ihrer Umgebung ab.

IV. Frl. Harmuth, wiederholt an Hornhaut-Fntzündungen gelitten, deren letzte um Weihnachten 1883 zum Abschluss kam; seitdem ist die linke Cornea erheblich getrübt.

5. Februar 1885. Status, 1. Auge. Cornea: gerade in ihrem Centrum präsentirt sich eine grauweissliche, schwach durchscheinende Trübung, die sich scharf von der umgebenden, normal spiegelnden und durchsichtigen Cornea abhebt und einen Flächendurchmesser von reichlich $3 \mathrm{Mm}$. hat.

5. Februar 1885. Visus, 1. Auge $=\frac{20}{200}$ (Snellen).

Jäger 7. 5 Zoll Entfernung.

6. Februar 1885̃. Aibtragung des Epithels und der oberflächlichen Cornealschichten in der Ausdehnung der getrübten Parthie. - Jodoform, Atropineinträufelungen, Verband, Bettrube.

7. Februar 1885. Patient klagt über Schmerzen im 1. Auge: Verbandwechsel. Das ganze Auge lebhaft injicirt, empfindet starke Lichtscheu bei reichlicher Thränensecretion; Cornea entsprechend den abgekratzten Parthieen 
gleichmässig tiefgrau getrübt. Ordo wie am 6. Februar 1885, dazu zwei Mal täglich warme Umschläge.

10. Februar 1885. Reizerseheinungen fast ganz geschwunden; es besteht eine starke Entwicklung von Blutgefässen, die zu dem frischen, noeh nicht mit Epithel versehenen Infiltrat hinziehen.

13. Februar 1885. Das Infiltrat hat an Intensität der Farbe sichtlich abgenommen, Epithel ersetzt, Reizerscheinungen geschwunden. Beginn der Massage.

20. Februar 1885. Visus, 1. Auge $=\frac{20}{70}$ (Snellen). Jäger 4. 5 Zoll Entfernung.

28. Februar 1885. Visus, l. Auge $=\frac{20}{70}$ (Snellen). Jäger 3. 5 Zoll Entfernung.

10. Mai 1885. Visus, l. Auge $=\frac{20}{70}$ beinahe $\frac{20}{50}$ (Snellen). Jäger 2. 5 Zoll Entfernung.

Entsprechend dieser Besserung des Visus war eine geradezu auffallige Aufhellung des Status zu constatiren; es lässt sich übrigens nicht leugnen, dass in diesem Fall: gleich nach der Operation sich eine Besserung des Status ergab, was auch durch die augenblicklich nach der Operation beobachtete Besserung des Visus von $\frac{20}{200}$ (Snellen) und Jäger 7 auf $\frac{20}{70}$ (Snellen) und Jäger 4 zu ersehen ist.

V. C. Hasenpusch, 26 Jahre alt, im December 1884 an einem Ulcus serpens der r. Cornea erkrankt, stellte sich am 5. Februar 1885 mit einer intensiven Trübung behufs Aufstellung derselben vor.

5. Februar 1885. Status, r. Auge. Cornea: in ihrer 
äusseren Hälfte ist sie Sitz einer intensiven, ca. $4 \mathrm{Mm}$. von rechts nach links langen, ca. $3 \mathrm{Mm}$. von oben nach unten breiten Trübung; dieselbe überlagert zum Theil das Pupillargebiet und ist intensiv weiss.

5. Februar 1885. Visus, r. Auge $=\frac{20}{100}$ (Snellen). Jäger 8. 10 Zoll Entfernung.

21. Februar 1885. Nach 16 maliger täglicher Massage hat die Trübung an Intensität deutlich abgenommen, besonders haben die peripheren Theile eine weniger intensive Färbung angenommen und sind durchscheinender geworden. In dem Centrum der Trübung ist die Aufhellung noch nicht zu constatiren.

21. Februar 1885. Visus, r. Auge $=\frac{20}{70}$ (Snellen).

Jäger 6. 6 Zoll Entfernung.

5. März 1885. Aufhellung jetzt auch in dem Centrum der Trübung leicht erkennbar.

5. März 1885. Visus, r. Auge $=\frac{20}{50}$ (Snellen).

Jäger 2. 6 Zoll Entfernung.

26. März 1885. Visus, r. Auge $=\frac{20}{40}$ (Snellen).

Jäger 2. 6 Zoll Entfernung.

1. April 1885. Visus, r. Auge $=\frac{20}{40}$ (Snellen).

Jäger 1. 6 Zoll Entfernung.

VI. A. Stein, 15 Jahre alt, als Kind häufig von scrophulösen Ophthalmieen heimgesucht, im Jahre 1882 an beiderseitigen Granulationen erkrankt. Die restirenden 1 Jahr alten Hornhauttrübungen beider Augen wurden Januar 1885 Gegenstand unserer Behandlung.

22. Januar 1885. Status, r. Cornea: oberflächliche, mattgrane, die Cornea beinahe völlig einnehmende, durch- 
scheinende Trübung: ihre Intensität ist insofern an den. verschiedenen Stellen versehieden, als die vor der Pupille gelegenen Parthieen am intensivsten trübe sind.

L. Cornea: gleichmässig, diffus mattgrau getrübt, aus dieser gleichmässigen Trübung hebt sich von der Mitte der Hornhaut nach unten innen nach der Corneoseleralgrenze ziehend eine intensivere weissgraue Trübung $a b$, ca. $3 \mathrm{Mm}$. lang, ca. $1 \mathrm{Mm}$. breit.

22. Januar 1885. Visus: beide Augen zahlen Finger anf beinahe 20 Fuss Entfernung. - Beginn der Massage.

23. Februar 1885. Visus, r. Auge $=\frac{20}{100}$ (Snellen). Jäger 13. 4 Zoll Entfernung.

23. Februar 1885. Visus, l. Auge $=\frac{20}{100}$ (Snellen). Jäger 14. 5 Zoll Entfernung.

5. März 1885. Visus, тr. Auge $=\frac{20}{70}$ (Snellen). Jäger 13. 4 Zoll Entfermung.

5. März 1885. Vitus, 1. Auge $=\frac{20}{100}$ (Snellen). Jager 7. 5 Zoll Entfernung.

Die Kranke giebt an, einen entschieden bedeutenden Fortschritt in der Sehschärfe an sich zu constatiren; die Trübungen haben sich eigentlich an Intensităt und Färbung nicht viel geändert.

VII. E. Schlömp, 18 Jahre alt, seit 4 Jahren bestehen rechts intensive Hornhautflecken.

4. Februar 1885. Status, rechte Cornea in ihrem Centrum intensiv weiss gefärbt in einem Wlächendurchmesser von ea. $2^{1 / 2} \mathrm{Mm}$.; nach innen davon ein punktförmiger, mattgrauer Hornhautfleck. 
4. Februar 1885. Visus, r. Auge $=$ Finger gezählt auf 7 Fuss Entfernung. Jäger 15. 3 Zoll Ėntfernung.

Beginn der Massage.

20. Februar. 1885. Visus, r. Auge $=$ Finger gezählt auf 15 Fuss Entfernung. Jäger 8. 3 Zoll Entfernung.

26. Februar 1885. Visus, r. Auge $=$ Finger gezählt auf 20 Fuss Entfernung. Jäger 5. 5 Zoll Entfernung.

12. März 1885. Visus, r. Auge $=\frac{20}{200}$ (Snellen). Jäger 3. 6 Zoll Entfernung.

31. Mărz 1885. Visus, r. Auge $=\frac{20}{200}$ (Snellen). Jäger 3. 6 Zoll Entfernung.

Obige Daten ergaben eine constant fortschreitende Besserung des Sehvermögens und man sollte eigentlich auch eine dementsprechende Aufhellung der Hornhauttrübungen erwarten. Von einer Aufhellung war aber absolut nicht die Rede, im Gegentheil, die Trübung präsentirte sich am Schlusse der Behandlung genan so wie am Anfange. Wie dieser Widerspruch zwischen der Besserung des Visus und der thatsächlich unveränderten, keineswegs geförderten Hornhąuttrübung zu erklären ist, vermag ich nicht hinreichend zu beleuchten.

VIII. Frl. Schwarplies stellte sich im März 1884 in der Heisrath'schen Poliklinik mit folgendem Status vor: rechtes Auge zeigt eine naeh Granulationen entstandene totale Versehwärung der inneren Hornhauthälfte, sodann eine etwas oberbalb der Mitte des unteren Hornrandes liegende Perforation; Visus: in Entfernung von einigen Fuss ca. wird Hell und Dunkel untersehieden. Nach Excision der Granulationen nach Heisrath'scher Methode war Patientin bereits in 6 Wochen von ihren Granulationen befreit und die Corneo erhalten; am 
30. Januar 1885 stellt sie sich behufs Anfhellung der restirenden Hornhauttrübungen von Neuem vor.

30. Januar 1885. Status, r. Auge: Cornea in ihrem unteren inneren Quadranten durchweg, besonders intensiv im unteren Theil, weissgraulich getrübt; leichtere Trübungen liegen im Hornhautcentrum und im oberen, äusseren Quadranten. Pupille unten adhärent, im Pupillargebiet besteht ein Exsudat, dessen obere Parthie nur wenig intensir gefürbt, mehr diffus getrübt erscheint, dessen untere Parthie dagegen intensiv getrubt, weiss gefärbt ist.

30. Januar 1885. Visus, r. Auge $=$ Finger gezählt auf 20 Zoll Entfernung. Jäger 14 in 5 Zoll Entfernung.

1. Februar 1885. Abkratzung des Hornhautepithels; in dem unteren, inneren Quadranten werden die obersten Cornealschichten mit abgeschabt; 10 Tropfen 3procentiger Cocainlösung ergaben eine genügende Anaesthesie. Die NachbehandIung geschah wie gewöhnlich, die anfangs sehr heftigen Reizerscheinungen lassen 4 Tage post operationem völlig nach, das Epithel ist am 6. Tage ersetzt.

7. Eebruar 1885. Beginn der Massage, anfangs einen Tag um den anderen, nach 14 Tagen tâglich.

6. März 1885. Visus r. Auge $=$ Jäger 11.6 Zoll Entfernung.

20. März 1885. Visus, r. Auge $=$ Jäger $10.6 \mathrm{Zoll}$ Entfernung. $\frac{20}{200}$ (Snellen).

Leider fehlt die Prüfung mit den Snellen'schen Tafeln vom 6. Marz 1885; soweit als die Angaben der Patientin hier gelten können, theile ich mit, dass sie sicher angab, bei der Prüfung am 6. März 1885 noch nicht die erste Reihe der Snellen'schen Tafeln gesehen zu haben. 
4. April 1885. Visus, r. Auge $=\frac{20}{200}$ (Snellen).

Jäger 7. 5 Zoll Entfernung.

Die "post operationem eingetretene frische Cornealinfiltration war anfangs in Ausdehnung und Farbe sehr intensiv, hat sich aber im Verlaufe der Behandlung entschieden sehr aufgehellt.

Die Massage wird in der Heimath der Patientin fortgesetzt.

IX. Eisenstadt, 22 Jahre alt, vor 10 Jahren an beiderseitigen Bindehautgranulationen erkrankt, hat seit 4. Jahren unverändert bestehende Hornhauttrübungen.

27. Februar 1885. Status, r. Auge. Cornea: diffuse, mattgraue, die ganze Cornea als leichte Wolke - mit Ausnabme des inneren, unteren Quadranten - einnehmende Trübung.

L. Auge. Cornea: desgleichen diffuse, völlig durchscheinende Trubbung der ganzen Cornea, daneben verschiedene stärkere, punktförmige Flecken besonders innen und oben aussen gelegen.

27. Februar 1885. Visus, r. Auge $=\frac{20}{100}$ (Snellen). Jäger 4. 5 Zoll Entfernung.

27. Februar 1885. Visus, l. Auge $=\frac{20}{100}$ (Snellen). Jäger 3. 6 Zoll Entfernnng.

Beginn der Massage.

5. März 1885. Weder im Status noch im Visus irgendwo ein Fortschritt za constatiren.

15. März 1885, Visus, r. Auge $=\frac{20}{100}$ (Snellen). Jäger 2. 5 Zoll Entfernung. 
15. März 1885. Visus, l. Auge $=\frac{20}{100}$ (Snellen).

Jăger 2.8 Zoll Entfernung.

31. März 1885. Status et Visus idem.

Der Erfolg der Massage war in diesem Falle ein ziemlich negativer; übrigens war Patient schon lange Zeit vorher mit den versehiedensten Aufhellungsmitteln olne Effect behandelt.

X. M. Römer, seit mehreren Jahren an beiderseitigen Granulationen leidend, welche durch die Excision der granulös veränderten Bindehautparthieen geheilt wurden, stellt sich zur Aufhellung ihrer am rechten Auge restirenden Hornhauttrübungen vor.

2. März 1885. Status r. Auge. Cornea: Dieselbe ist Sitz einer totalen Trübung von mattgrauer Farbe; innerhalb derselben bestehen vereinzelt sich abhebende, weisslich graue, punktförmige Trübungen und eine das Pupillargebiet z. Th. bedeckende Trübung von ca. $2 \mathrm{~mm}$ Flächendurchmesser.

2. März 1885. Visus, r. Auge = Finger gezählt auf 3 Fuss Entfernung. Kein Jäger.

3. März 1885. Abtragung des Hornhautepithels and der obersten Hornhautsehichten fast in der Ausdehnung der ganzen Hornhaut bei localer Anaesthesie dureh 3pCt. Cocain. Behandlung wie gewöhnlich.

5. März 1885. Sehr heftige Reizerscheinungen und Schmerzen im Auge; Cornea völlig, diffus mattgrau getrübt, entbehrt ganz des Epithels.

12. März 1885. Schmerzen nicht mehr vorhanden, jedoch ist das Auge noch immer lebhaft geröthet und lichtscheu. Die frisch infilttirte Cornea ist stark vaseularisirt.

22. März 1885. Beginn der Massage: Reizerscheinungen geschwanden, Epithel völlig ersetzt. 
Visus, 25. April 1885. Patient $=$ zählt Finger auf 12 Fuss Entfernung.

Jäger 20 in 5 Zoll Entfernung.

Die Massage wird fortgesetzt.

Der Verlauf der Operation war in diesem Falle ein ziemlich schwerer und protrahirter, was wohl nicht wunderbar erseheint, wenn man bedenkt, dass die Cornea total ihres Epithels und ihrer oberen Schichten entledigt wurde. -

Bevor ich noch einige kurze Worte über die Theorie der Wirkungsweise der Massage bei den Hornhauttrutbungen sage, bemerke ich noch, dass die vorgelegten Falle von Hornhauttrübungen beinahe sämmtlich alte und auch meist sehr umfangreiche Trübungen darstellten und somit ohne Ausnahme eine für die eventuelle Aufhellung wenig günstige oder sogar völlig ungünstige Prognose im allgemeinen stellen liessen. Trotzdem, glaube ich, konnte ich im Vorstehenden darthun, dass auch in solchen Fallen die Massage noch recht gutes zu leisten vermag. Es bedarf aber stets - zumal bei so alten Trübungen - einer ziemlich langen Anwendung der Massage, wenn man Erfolge zu verzeichnen haben will. - Was nun die Art der Massagewirkung beim Auge betrifft, so lässt sich darüber speciell allzuviel neues nicht sagen, sondern es handelt sich wohl um eben das, was die Massage auch an anderen Stellen leistet. Den einen der von mir mitgetheilten Fälle vermag ich nicht genügend zu erklären in seinem Widerspruch zwisehen Visus und Status, die anderen meiner Falle liegen viel einfacher, denn wo überhaupt ein Heilerfolg zu constatiren war, ergab die Untersuchnng auch ein Hellerwerden der Trübungen. Das Zustandekommen dieser Aufhellung haben wir uns wohl so zu erklären, dass die Massage des Anges sr ein mechanisch depletorisch wirkend, angehäufte Exsudatmassen und organiirte Entzündungsproducte der Hornhaut zerreibt und ఛ. Graefe's Archiv für Ophthalmologie, XXXI. 3. 
direet in die Oeffnungen der für die Flüssigkeitsaufsaugung bestimmten Bahnen bineindrückt. Daneben wirkt aber auch die Massage - wie jedes andere Aufhellungsmittel für Hornhauttrübungen - als directes Reizmittel und bahnt so die Reorption von Exsudatmassen an. Wir stellen uns vor, dass in Folge des gesetzten Reizes eine Vermehrung der Stoffzufuhr, eine Hyperämie erzielt wird, dass sich auf solche Weise eine Aufweichung der neugebildeten Elemente ergiebt, welche der Zerfällniss der Elemente und deren Resorption sehr günstig Vorschub leistet.

So erklüren wir uns leicht und plausibel die Massagewirkung am Auge und werden sie gerne als eine sehr rationelle Heilmethode auch für die Augenheillunde anerkennen. 